\title{
Tactile Guides for Touch Screen Controls
}

\author{
Robert Kincaid \\ Agilent Laboratories \\ 5301 Stevens Creek Blvd. MS4U-SC \\ Santa Clara, California 95051 \\ robert_kincaid@agilent.com
}

\begin{abstract}
While multi-touch devices such as smart phones and tablets offer simple intuitive user interfaces, such interfaces may not always be optimal for precision tasks typical of electronic measurement instruments. Such instruments utilize physical knobs, switches and sliders to provide precise tactile control of instrument parameters and states. This paper describes work-inprogress to investigate the use of simple transparent overlays that provide tactile guides for touch-based graphical control widgets. By augmenting the touch surface with a simple low-cost overlay, we aim to restore many of the tactile properties and benefits of standard physical controls. We have fabricated an initial proof-of-concept overlay and demonstration system and have performed a preliminary evaluation that suggests overlay guides may be beneficial.
\end{abstract}

Touch screen. Tactile. Tangible. Overlay. Instrument control. Multi-touch. Physical interface.

\section{INTRODUCTION}

Complex scientific and electronic measurement instruments often require precise adjustments which are typically accomplished with physical controls such as buttons, knobs, sliders and switches (for example see Figure 1). Users of modern test equipment are accustomed to these physical controls, which by learning and adaptation are perceived as natural and intuitive. More importantly, physical control surfaces allow for precise, intuitive manipulation while the operator's vision is cognitively focused on the visual task at hand rather than on the control device. A typical example would be an oscilloscope user adjusting a wave form display via adjustment knobs. More commonly, anyone who has compared a physical versus an on-screen keyboard will quickly grasp the significant benefit of physical vs. virtual input devices for some tasks.

In recent years touch screen interfaces have emerged as a popular, flexible and intuitive means of human computer interaction. For example, zoom operations can be accomplished quite intuitively using pinch gestures and panning operations by touch-and-drag movements. In contrast, there is evidence that for some precision tasks, touchbased graphical analogues to knobs and sliders are generally less effective than their physical counterparts (Crider et al., 2007). Despite these taskspecific usability issues, touch-enabled devices such as smart phones and tablets have become ubiquitous and users of test and measurement equipment will become increasingly accustomed to the affordances of intuitive multi-touch interfaces.

Thus, we were led to explore the design possibilities that modern touch screens and portable devices might offer test and measurment instruments. Toward this end, this paper presents work-in-progress that specifically examines the use of static overlays used in conjunction with touch screens to provide tactile guides. We use these guides hoping to offer the user some of the same tactile attributes of physical controls while maintaining the overall compactness and intuitiveness of portable touch screen devices. In particular, we aim to facilitate locating and operating controls by feel rather than sight, bridging

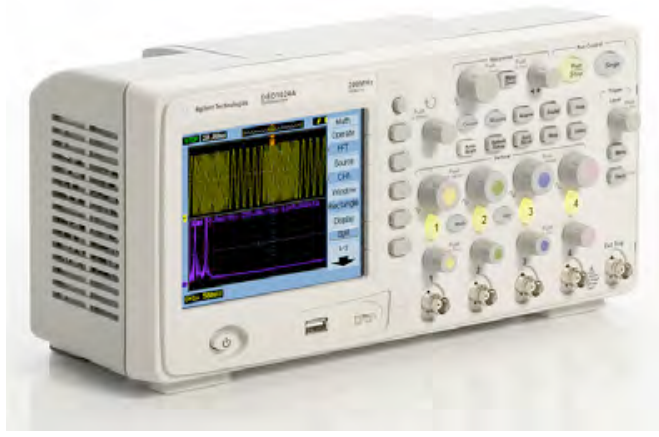

Figure 1. A typical small portable oscilloscope with an analytical display area on the left and a dedicated control surface on the right consisting of a variety of knobs and push buttons. 
the gap of traditional physical instrument controls with more tactile touch-based counterparts, and thereby hope to leverage the best aspects of both styles of interaction. Since such instruments are dedicated to particular tasks (in contrast to a general purpose tablet or computer) the static nature of such overlays is an acceptable and potentially preferred design choice that mimics the familiar static layout of conventional instruments.

Contributions of this work-in-progress are:

- A prototype system demonstrating how a tablet computer and tactile guides can support an oscilloscope-like instrument.

- A preliminary evaluation that suggests such guides may be beneficial.

- Insight into how users interact with such controls and observations on what benefits tactile guides may afford.

\section{RELATED WORK}

The recent advent of multi-touch interfaces has opened up a number of intriguing possibilities for more natural and intuitive interfaces. However, for certain specialized tasks, physically graspable controls provide superior fine motor control and more direct manipulation. Fitzmaurice and Buxton (Fitzmaurice and Buxton, 1997) showed that for some target tracking tasks, dedicated physical devices with specific shapes and function were more effective than generic input devices. In particular they note that it "may be faster to acquire an attached device that is out of hand than to attach to virtual controls with a device in hand." Swindells, Tory, and Dreezer (Swindells et al., 2009) compared the performance of mice, sliders and pen input. They found that physical sliders required significantly fewer visual fixations to operate the control, suggesting that this frees the user's visual attention for more efficient task-specific cognition. From expert reviews of a haptically enhanced mixing board, Crider et al. (Crider et al., 2007) found a similar result for graspable sliders. This work also considered bimanual operation. They found that the graspable nature of the physical sliders permitted two-handed operation although users seldom performed simultaneous two-handed operations.

Much of the work in augmenting touch screens relating specifically to tactile guides has been focused on efforts to assist individuals with sight impairment, often using some form of overlays (Challis and Edwards, 2001, Landau and Wells, 2003). It should be noted that the design goals in such cases are substantially different from those for visually intensive tasks such as operating a test and measurement device. For example, with sight impairment the main goal is generally to augment visual user interfaces with tactile replacements.
However, for some complex tasks using normal vision, the goal may be to minimize the need to visually fixate on operating the control in order to free the user to visually focus on the real-time results of manipulating the control (e.g. controlling the waveform display on an oscilloscope). Also, overlays intended for the visually impaired strive to make as much of the interface accessible as possible by using tactile cues. In contrast, designing for a measurement instrument using tangible augmentation, only a portion of the display need be dedicated to a tactile control surface and the remaining portion of the display is still used as a standard visual data display. This non-control region may even be responsive to standard touch screen gestures that augment functions of the dedicated control surface.

We are also informed by the general research in tactile interfaces. For a brief review of recent approaches see (Benali-Khoudja et al., 2004). A related area of research strives to dynamically deform a touch screen surface in order to provide dynamic tactile control surfaces. While these systems are promising, they generally rely on complex electronic (Bau et al., 2010) and/or mechanical (Harrison and Hudson, 2009) systems and have not yet seen wide adoption beyond the research lab. Another technique is to re-introduce tangible controls to a touch screen through physical devices that interact directly with the screen itself such as Clip-On Gadgets (Yu et al., 2011), CapWidgets (Kratz et al., 2011) or Spinner (Kobayashi and Akamatsu, 2005).

DataTiles (Rekimoto et al., 2001), consist of transparent tiles used for small tangible information displays and controls using an underlying flat panel display to label and visualize the contents of each tile. Grooves are used to guide pen input rather than finger touch and to implement rudimentary pen-based tangible controls. Casiers (Ullmer et al., 2011) introduced guided control surfaces using "slotted widgets" that provided tactile guides for touch based control panels. However, Casiers did not focus on integrating precision touch-based control widgets and an analytical display within the same device or consider issues related to instrument design. There was also no comparison of user performance with and without tactile guides.

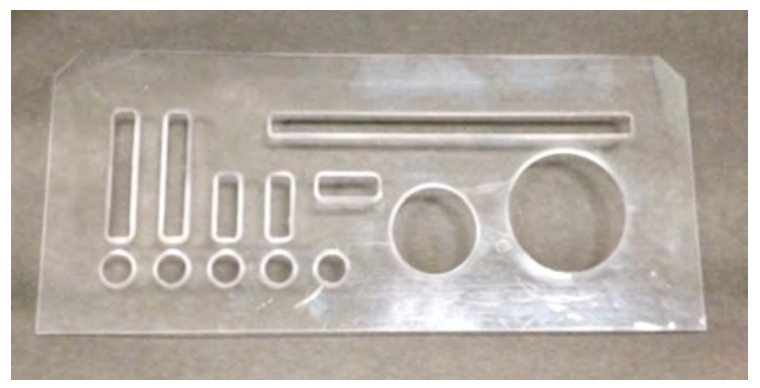

Figure 2. Prototype Lexan overlay. 


\section{PROOF-OF-CONCEPT PROTOTYPE}

Our prototype uses software designed for an Apple iPad 2 and displays real-time waveforms of live audio received by the built-in microphone. The software can display sampled waveforms, a frequency spectrum or a spectrograph. Our intent is to provide a proof-of-concept demonstration using a readily available platform in order to mimic some of the interactions required of an oscilloscope or spectrum analyzer and thereby provide a simple surrogate for a full instrument. The overlay was fabricated from a sheet of clear Lexan 0.0625 in (1.5875 $\mathrm{mm}$ ) thick. This material is transparent and easy to cut and machine as required. Figure 2 shows the overlay alone while Figure 3 shows the overlay mounted on an Apple iPad 2 along with the prototype software. During testing the overlay is merely secured temporarily with electrical tape. In an actual instrument we envision the overlay would be permanently mounted and finished to look like an integral part of the device. Such permanent mounting is consistent with the concept of building a dedicated measurement instrument with a fixed control area and a fully touch-enabled display area. The overlay is of sufficient thickness that touch events are only detected within the cut-outs, where direct contact with the touch screen is possible.

For prototyping purposes, sliders and switches in a variety of orientations as well as buttons and dials are implemented as custom user interface compo- nents. We designed our first overlay with a generic sampling of various control types without regard for final function. In the instrument prototype we simply map many of these controls to appropriate functions for controlling the waveform display and therefore not all controls currently have an assigned function. Buttons are used to select between various display options. Dial controls are used to adjust the overall gain and the baseline offset of the signal display. Finally a slider is used to set the sampling interval in milliseconds and a switch enables/disables gestures.

Since the overlay is transparent we can create dynamic labels and tic marks as with a traditional touch-only display. This allows changes in colour to indicate whether or not a two-state button is pressed. Unlike static physical controls, we can dynamically move labelling with the movement of the control. For instance, in Figure 3, the large horizontal slider shows the numerical setting above the slider position, and this tracks with the slider as it is moved. This same setting can also be achieved by a simple pinch gesture on the waveform display. In this case the slider itself adjusts automatically in real-time to reflect the pinch-induced setting, including tracking the label with the slider control. Such synchronization is only achievable with physical controls by relatively complex motorized control surfaces (e.g. high-end recording studio mixing boards).

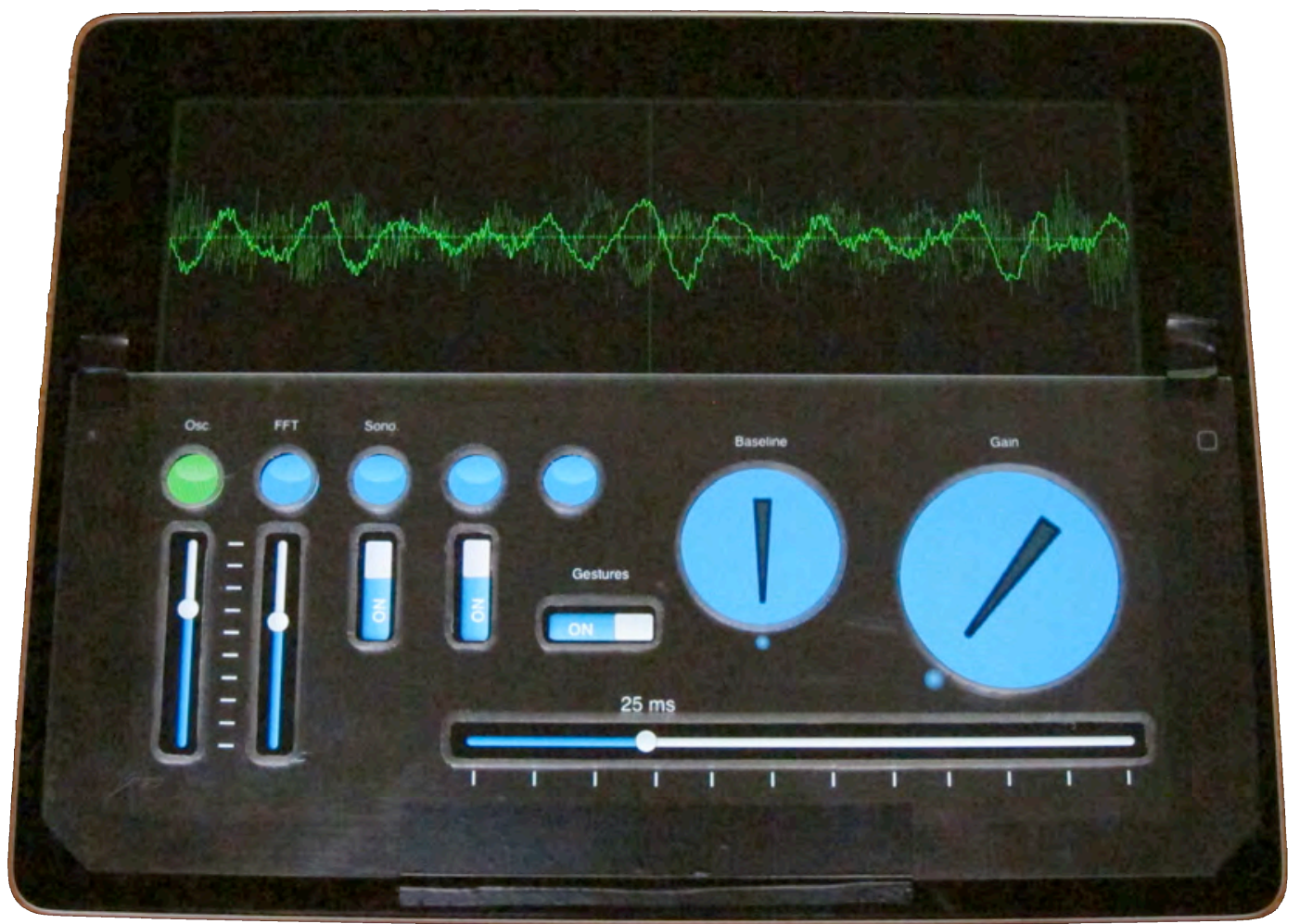

Figure 3. The complete prototype system. The upper portion of the screen shows a live waveform while the bottom area includes the overlay and various control widgets. Control labels and tick marks are rendered by the touch screen. 


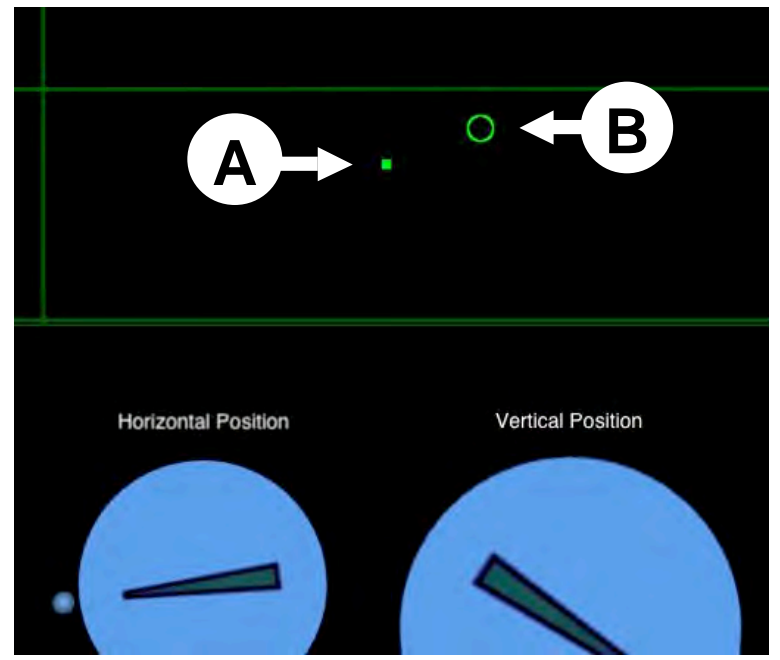

Figure 4. Example of the target seeking navigation task. Test subjects were required to move cursor $(A)$ inside target $(B)$ using the two dials shown for horizontal and vertical movement.

\section{PILOT EVALUATION}

We conducted a very preliminary pilot evaluation using a simple target seeking navigation task utilizing the two dial controls. Eight subjects ( 5 males, 3 females) were recruited from Agilent Laboratories ranging in age from 28 to 56 with an average age of 41 . All subjects were highly trained researchers with experience in measurement equipment as well as previous exposure to touch screen devices.

The within-subjects design required navigating a cursor from the center of the display area to a designated target using the two dial controls for separate horizontal and vertical positioning (see Figure 4). After a brief orientation and practice run, each subject was presented with six different target positions in sequence. These target positions were constrained to be the same distance from the initial cursor position at the center of the screen. This sixtarget scenario was repeated twice with and twice without the overlay for a total of 24 individual measurements per subject. Half of the subjects began with a bare touch screen and half began with the overlay in order to compensate any potential biases from learning effects.

This task was not intended to reflect the most effective means of $x / y$ navigation on a touch screen. Clearly, simply touching the target directly would lead to a more immediate result (although perhaps with some loss of precision). However, the goal in this instance was to specifically examine the potential improvement in performance that tactile guides might provide for virtual dials. We desired a simple rudimentary task of moderate complexity requiring fine motor control and suitable for controlled measurement. Note that we began our investigation with dials based on the hypothesis that these controls would likely see the most benefit from tactile guides.

Figure 5 presents a summary of these measurements. We analyzed total task completion time, summing the individual times for each subject under each condition. These preliminary results support the hypothesis that task completion time tends to decrease when using the overlay. A paired t-test yielded a score of $t=2.54359$. Despite the relatively small sample size, the corresponding $p$-value of 0.03846 indicates a moderate statistically significant effect $(p<0.05)$. This result is encouraging that such overlays may provide benefit. A more thorough study with a larger number of participants would be required to make any conclusive claims.

It is notable that not all subjects saw a benefit in task performance. Three of the subjects saw either small benefit or even slightly increased task completion times when using the overlay. However, a majority of the subjects $(5 / 8)$ did obtain decreased completion times when using the overlay.
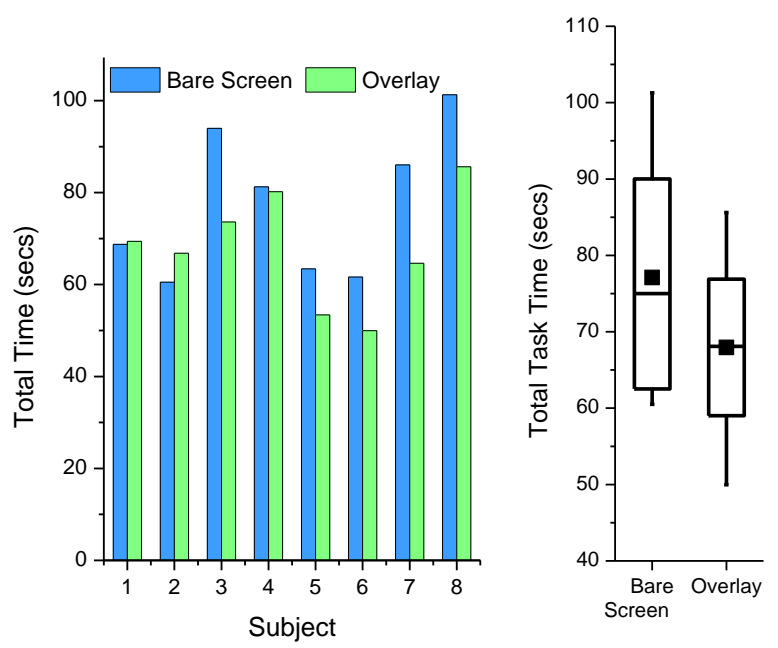

Figure 5. Sumary of total task completion times measured by the pilot study. The bar chart compares each subject with and without the overlay in place. The box plot summarizes the mean (black square) and statistical variance between subjects with and without the overlay.

Several subjects in the evaluation were observed avoiding the edge of the dial cutouts and operated the dial freely, more-or-less as they did without the overlay. Two of the three subjects who did not see a benefit from the overlay were observed avoiding the edges in this manner. These two subjects were simply operating the dial in the same fashion whether the overlay was present or not. We plan to investigate implementing a dial control using a circular track vs. the current circular hole in order to constrain the user to operate within the confines of a tactile edge. 


\section{DISCUSSION}

While tracing the edge of a dial's circular cutout with one's finger, it is possible to sense the changes in curvature and the area of the finger that is in contact with the edge. This allows the user to detect on which point of the circle their finger resides. More simply, if one imagines the cutout corresponds to a clock face, it is relatively easy to know if one's finger is at 12 o'clock or 6 o'clock, etc. Even with sliders and switches one can sense by touch the extreme positions of these controls. Sensing this information non-visually is not possible with a bare touch screen. These observations support the idea that we are restoring significant tactile cues and also hint at the possibility that guided dials would provide more information than guided slider controls since dial cutouts presumably provide more perceptible intermediate position information.

Another advantage observed about tactile dial overlays is that it allows the user to take full advantage of the largest possible arc when manipulating the dial. Without guides, the user may have a tendency to work within the interior of the dial to avoid losing contact with the dial widget. In this case small, potentially non-circular movements can result in large and often inconsistent effects. Touches around the outer edge guide all share the same radial trajectory resulting in a more consistent response similar to that expected of a physical device.

Since the overlay thickness blocks sensing touch events and the user can operate controls by feel, bimanual operation is easily achieved on the touch screen surface by simply resting the appropriate left and right hand fingers inside the corresponding

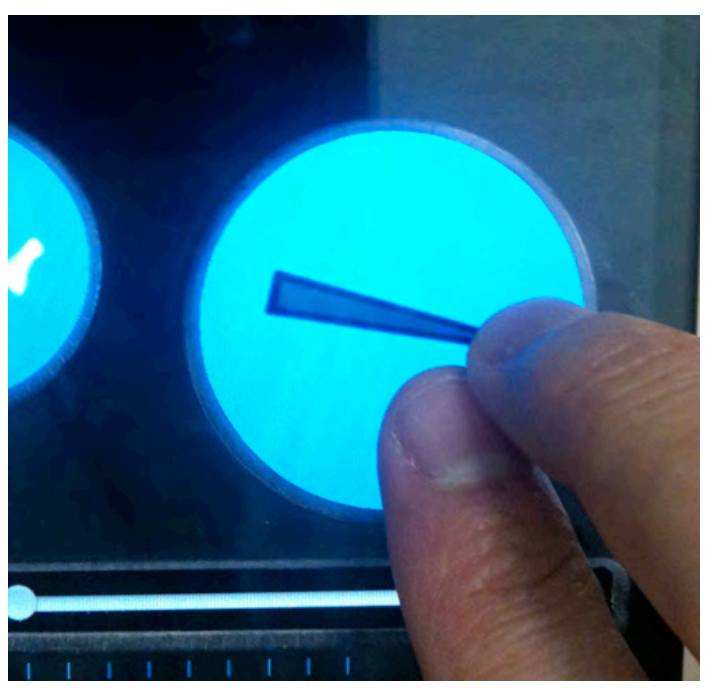

Figure 6. Example of two-finger gesture to operate a fineadjustment on the dial. controls and operating them solely by feel. Issues of palm and wrist contacts generating spurious events are essentially eliminated as well as difficulties in maintaining proper finger alignment with the control. Also, the user is no longer required to maintain awkward wrist postures to avoid erroneous palm and wrist contacts. We noted in the evaluation that several users spontaneously invoked bimanual operation and one user even manipulated both dial controls simultaneously to effect diagonal movement.

Open guides as designed in the current prototype fully expose the touch surface within the interior of the control. Therefore, it is still feasible to support touch-based gestures or other augmented interactions not possible with traditional physical controls such as a potentiometer. For example, we implemented a tap gesture within the dials to reset them to a default position. We can also recognize two finger touches as driving a "fine adjustment" as shown in Figure 6. In this case finger position is not mapped one-to-one with dial movement and is instead used to make smaller incremental movements of the dial.

The main drawback with attaching a fixed control surface to the touch screen is the loss of flexibility over a bare touch screen. This is offset somewhat since the function and labeling of control widgets can be easily changed at run time to support whatever task context is required. However, the physical layout of controls is fixed by the static overlay. Fortunately, for test and measurement equipment control surfaces are generally fixed and usually have minimal flexibility in terms of task-specific function. The static nature of control layouts in test and measurement equipment is a desired feature since it affords better user recall and kinesthetic awareness of control locations and functions.

As mentioned previously in Section 2, it is possible to create tangible controls that interact directly with a touch screen surface either electrically as with Spinner (Kobayashi and Akamatsu, 2005), via capacitive sensing (Kratz et al., 2011, Yu et al., 2011), or optically as implemented in the Reactable (Jordà et al., 2007). Such controls could be constrained within an overlay to permit actual physical controls to be operated in a robust static layout while still using the touch screen as the underlying sensing technology. This is somewhat similar conceptually to the "Token+Constraint" concept (Ullmer et al., 2003) and embodied in Casiers (Ullmer et al., 2011). This might have advantages in lower manufacturing cost over electronic control panels, and minimize the necessity for supporting the electronics and electrical connections required by traditional electronic control devices. 


\section{CONCLUSIONS AND FUTURE WORK}

This paper presents very preliminary results of a work-in-progress that explores the use of tactile guides on touch screen displays in the context of test and measurement instruments. Initial feedback from demos and live use as well as the results of our preliminary pilot evaluation study are encouraging that this approach may provide significant tactile benefit, replacing some of what is lost in moving from physical controls to a touch-only system. We plan to conduct more thorough and conclusive user studies to evaluate the degree of improved usability these overlay guides provide, and with which specific user interface widgets and tasks they are most beneficial. We are also interested in exploring how overlays can be further augmented with tangible controls and what benefits and tradeoffs exist between bare displays, simple tactile guides and more complex but more realistic tangible control panels.

While our current prototype is focused on electronic test and measurement, we speculate other devices with dedicated control surfaces might benefit from such tangible guides (cameras, household appliances, automotive control surfaces, etc.).

No circular cutout will ever replace the tactile sensation and exquisite control of a finely crafted physical knob. However, it is our hope that for test and measurement instruments, such guides can provide substantial improvement over bare touch screens at very low cost and simple overall design while still leveraging many of the benefits offered by modern multi-touch interfaces.

\section{ACKNOWLEDGMENTS}

We thank Diane Larson, Michael Domler and Randy Fehlhafer from the Agilent Model Shop for their assistance in fabricating our initial overlay.

\section{REFERENCES}

Bau, O., Poupyrev, I., Israr, A., et al. 2010. TeslaTouch: electrovibration for touch surfaces. Symp. on User Interface Software and Technology. New York, New York, USA: ACM.

Benali-Khoudja, M., Hafez, M., Alexandre, J. M., et al. 2004. Tactile interfaces: a state-of-the-art survey. Int'l Symposium on Robotics.

Challis, B. P. \& Edwards, A. D. N. 2001. Design principles for tactile interaction. Haptic HumanComputer Interaction.

Crider, M., Bergner, S., Smyth, T. N., et al. 2007. A mixing board interface for graphics and visualization applications. Graphics Interface. Montreal, Canada: ACM.

Fitzmaurice, G. W. \& Buxton, W. 1997. An empirical evaluation of graspable user interfaces: towards specialized, space-multiplexed input. SIGCHI Conf. on Human Factors in Computing Systems. Atlanta, Georgia, United States: ACM.

Harrison, C. \& Hudson, S. E. 2009. Providing dynamically changeable physical buttons on a visual display. SIGCHI Conf. on Human Factors in Computing Systems. Boston, MA, USA: ACM.

Jordà, S., Geiger, G., Alonso, M., et al. 2007. The reacTable: exploring the synergy between live music performance and tabletop tangible interfaces. TEl'07. ACM.

Kobayashi, S. \& Akamatsu, M. 2005. Spinner: a simple approach to reconfigurable user interfaces. Proceedings of the 2005 conference on New interfaces for musical expression. Vancouver, Canada.

Kratz, S., Westermann, T., Rohs, M., et al. 2011. CapWidgets: tangbile widgets versus multi-touch controls on mobile devices. Extended Abstracts SIGCHI Conf. on Human Factors in Computing Systems ACM.

Landau, S. \& Wells, L. 2003. Merging tactile sensory input and audio data by means of the Talking Tactile Tablet. EuroHaptics' 03.

Rekimoto, J., Ullmer, B. \& Oba, H. 2001. DataTiles: a modular platform for mixed physical and graphical interactions. SIGCHI Conf. on Human Factors in Computing Systems ACM.

Swindells, C., Tory, M. \& Dreezer, R. 2009. Comparing Parameter Manipulation with Mouse, Pen, and Slider User Interfaces. Computer Graphics Forum, 28, 919-926.

Ullmer, B., Dell, C., Gil, C., et al. 2011. Casier: structures for composing tangibles and complementary interactors for use across diverse systems. TEl'11. Funchal, Portugal: ACM.

Ullmer, B., Ishii, H. \& Jacob, R. 2003. Tangible query interfaces: Physically constrained tokens for manipulating database queries. Interact'03. Zurich, Switzerland.

Yu, N.-H., Tsai, S.-S., Hsiao, I.-C., et al. 2011. Clipon gadgets: expanding multi-touch interaction area with unpowered tactile controls. Symp. on User Interface Software and Technology. Santa Barbara, California, USA: ACM. 